most important target volume dose parameter) was then investigated.

Results MR based assessment of tumor volumes initially, at the time of the first and the fourth BRT application, were as follows: $(49.9 \pm 33.3 \mathrm{ccm}, 11.3-124.2 \mathrm{ccm}),(17.3 \pm 19.2 \mathrm{ccm}$, $1.7-78.4 \mathrm{ccm})$ and $(7.0 \pm 10.9 \mathrm{ccm}, 0.8-58.5 \mathrm{ccm})$ respectively. Registered EQD2 HR-CTV doses for the whole group of patients were D90 (107.15 $\pm 22.06 \mathrm{~Gy})$, and D100 (80.66 $\pm 14.58 \mathrm{~Gy})$. Initial tumor volume showed a strong negative, statistically significant correlation with registered HR-CTV D90 dose at the time of the first BRT application (rho= 0.8 ). ROC analysis confirmed the discriminative influence of initial tumor volume on achieving recommended HR-CTV dose, with the best initial tumor volume cut-off value of $47.87 \mathrm{ccm}$, with high sensitivity $89.4 \%$, and specificity $90.9 \%$. A statistically significant difference between two groups of patients (regarding the identified tumor volume cut-off value of $47.87 \mathrm{ccm}$ ) was found for the EQD2 HR-CTV doses: D90 (120.4 $\pm 14.6 \mathrm{~Gy}$ vs $87.33 \pm 15.48 \mathrm{~Gy}, \mathrm{p}=6.9 * 10-7)$ and D100 $(101.8 \pm 13.39$ vs $75.85 \pm 11.93 \mathrm{~Gy}, \mathrm{p}=9 * 10-5)$.

Conclusion The initial volume of the cervical tumor has a great influence on achieving the recommended values of HRCTV D90 and D100 doses. Tumors with initial volume greater than $47.87 \mathrm{ccm}$ cause a statistically significant lower total EQD2 dose at the HR-CTV volume, leading to poor local disease control.

Disclosures The results are part of my paper published recently in $\mathrm{J}$ BUON.

https://jbuon.com/archive/25-1-108.pdf

\section{DISEASE-FREE SURVIVAL IN EARLY-STAGE CERVICAL CANCER: COMPARISON OF LAPAROSCOPIC VERSUS OPEN SURGERY IN A TERTIARY CENTRE}

Amanda Veiga-Fernández, María López-Altuna, Ignacio Romero-Martínez, Elsa Mendizábal Vicente, Patricia Rincon Olbes, Santiago Lizarraga Bonelli.

\subsection{6/ijgc-2020-ESG0.11}

Introduction/Background Over the last years, minimally invasive surgery was advancing as the preferred approach in many cancer centres for the treatment of early-stage cervical cancer, as it offers advantages against open abdominal surgery in inhospital and short-term outcomes. However, results from a recent randomized trial suggested lower rates of disease-free survival in the follow-up of patients operated with the minimally invasive approach.

The aim of our study was to compare, in our tertiary centre, the rate of disease-free survival at 3.5 years between both surgical techniques.

Methodology Retrospective single-centre study including patients who underwent total laparoscopic radical hysterectomy (LRH) or open radical hysterectomy (ORH) due to early-stage cervical cancer (IA1-IIA1) between 2005-2017.

Results A total of 63 patients were included (39 LRH and $24 \mathrm{ORH})$. Baseline characteristics are described in table 1. Mean age was similar in both groups $47.5 \pm 13.3$ vs. 48.3 $\pm 12.6(\mathrm{p}=0.8)$. The prevalence of high-risk HPV was similar in both groups with a higher rate of prior conization in the LRH group $22(56.4 \%)$ vs. $5 \quad(20.8 \%) \quad(p=0.006)$. There were no significant differences between both groups in terms of parametrial involvement, histologic subtypes and stage of disease. In almost half of the patients in the LRH group the uterine mobilizer was used during surgery, with 1 case of uterine perforation. There were no significant differences at 3.5 years follow-up in terms of recurrences [LRH 4 (10.3\%) vs. ORH $2(8.3 \%)(p=0.8)]$ and overall death (being secondary to their oncological process in all cases), $2(5.1 \%)$ vs. 1 $(4.2 \%) \quad(p=0.87)$. Kaplan-Meier analysis revealed a similar rate of disease-free survival at 3.5 years in both groups: LRH $87.2 \%$ vs. $87.5 \%(p=0.95)$ (figure 1$)$. The rate of disease-free survival in patients in which the uterine mobilizer was used was $100 \%$.

We hypothesized that, in experienced hands and with appropriate patient selection, a minimally invasive approach via laparoscopic surgery can be as effective as conventional

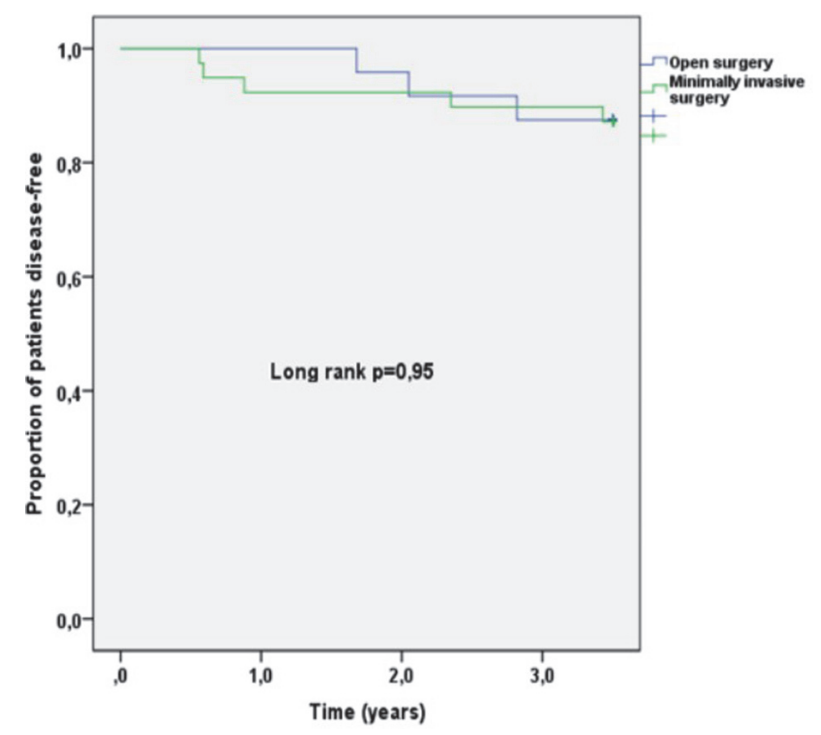

Abstract 154 Figure 1

\section{Abstract 154 Table 1}

\begin{tabular}{|l|l|l|l|}
\hline & $\begin{array}{l}\text { Laparoscopic radical } \\
\text { hysterectomy } \\
\mathrm{N}=39\end{array}$ & $\begin{array}{l}\text { Open radical } \\
\text { hysterectomy } \\
\mathrm{N}: 24\end{array}$ & \\
\hline Age (years) & $47,5 \pm 13,3$ & $48,3 \pm 12,6$ & $\mathrm{P}=0,8$ \\
\hline Prior conization & $22(56,4 \%)$ & $5(20,8 \%)$ & $\mathrm{P}=0,006$ \\
\hline High-risk HPV & $7(17,9 \%)$ & $1(4,2 \%)$ & $\mathrm{P}=0,2$ \\
\hline Parametrial involvement & $1(2,6 \%)$ & $2(8,3 \%)$ & $\mathrm{P}=0,3$ \\
\hline Histologic subtype & & $13(54,2 \%)$ & $\mathrm{P}=0,1$ \\
Squamous-cell carcinoma & $13(33,3 \%)$ & $6(25 \%)$ & \\
Adenocarcinoma & $13(33,3 \%)$ & $1(4,2 \%)$ & \\
Adenosquamous carcinoma & 0 & $3(12,4 \%)$ & \\
Other & $12(30,8 \%)$ & $1(4,2 \%)$ & \\
Not reported & $1(2,6 \%)$ & $7(29,2 \%)$ & \\
\hline Stage of disease & & $17(70,8 \%)$ & \\
IA1,IA2 & $14(35,9 \%)$ & 0 & \\
IB1,IB2 & $24(61,5 \%)$ & & \\
IIA1 & $1(2,6 \%)$ & $\mathrm{P}=0,6$ \\
\hline Use of uterine mobilizer & & 0 & \\
during surgery & & $24(100 \%)$ & \\
Yes & $18(47,4 \%)$ & 0 & \\
No & $9(23,7 \%)$ & $\mathrm{P}=1$ \\
Unknown & $11(28,9 \%)$ & $16(66,7 \%)$ & \\
\hline Uterine perforation during & $1(2,6 \%)$ & $6(25 \%)$ & \\
surgery & & $2(8,3 \%)$ & \\
\hline Adjuvant therapy & & & \\
No & $30(76,9 \%)$ & $7(17,9 \%)$ & \\
Radiotherapy & $2(8,3 \%)$ & & \\
Radiotherapy and & & & \\
Chemotherapy & & & \\
\hline
\end{tabular}


open surgery. However, the results must be interpreted with caution given the potential risk of bias derived from the relatively small sample size and the single-centre, retrospective nature of the study.

Conclusion In the present study, we did not find statistically significant differences between LRH and ORH for the treatment of early-stage cervical cancer in terms of disease-free survival and overall survival.

Disclosures Nothing to disclosure.

\section{CERVICAL ONCOLOGICAL CYTOLOGIES'S (PAP TEST) EVALUATION OF THE RIVERINE WOMEN FROM RIVERS TAPAJÓS AND CUPARI (PARÁ - BRAZIL)}

Maria Vitoria Lima Ferrero, Rafaela Estefano Uriza, Aline Silva Moreira Dos Santos, Letícia Bertaglia, José Anibale Rodrigues Junior, Jhenifer Nataly Moura França, Fabiana Moreira Passos Succi, Icléia Siqueira Barreto, Katia Piton Serra. São Leopoldo Mandic

\subsection{6/ijgc-2020-ESG0.12}

Introduction/Background Cervical cancer is the most prevalent in Brazil North Region. There are few studies about cervical injuries in the riverine women. The knowledge of these characteristics could improve health assistance to these women, contributing to health policies that benefit this population. Objective: This paper aims to evaluate the cervical oncological cytologies of the riverine women from rivers Tapajós and Cupari (Pará - Brazil).

Methodology A partnership between SLMandic Medical School and the local Cities of Santarém, Aveiro and Belterra (State of Pará, Brazil) Government was established trhough 'Barco da Saúde' (Health Boat) project. The Counties have provided a Hospital Boat named 'Abaré'. SLMandic has provided a team made up by professors and medical students. Services in pediatrics, gynecology and obstetrics, dermatology, ophthalmology and odontology were provided. In 2019, the activities were held between July 27nd and August 5th, 206 gynecological and obstetric appointments were made and 133 cervical oncological cytologies were collected. Epidemiological data were collected from anamnesis form designed specifically for the expedition. The reading of the cytology slides was performed and de injuries classified among high grade lesions (HSIL), low grade lesions (LSIL), scaly lesions of undetermined significance (ASC-US), and glandular lesions of undetermined significance (ASCH). Pathological agents found have also been described. The samples were classified between satisfactory and unsatisfactory. Finally, statistical analysis of data was done.

Results Out of 206 gynecological and obstetric appointments, $132(64.1 \%)$ cervical cytologies were collected. Among these patients, $100(75.75 \%)$ were screening age (25-65 years old). Median of patients age was 34.4 years old. About 128 (97\%) samples were satisfactory for analysis. Cervical cancer screening was the objective of the exam in 101 (76.5\%) of the cases. Related to associated microorganisms, Lactobacillus sp. were present in 68 (51.5\%), Gardnerella vaginallis in 13 (9.8\%), Candida sp. in $8(6 \%)$ Trichomonas vaginallis in 2 $(1,5 \%)$, in addition to others less prevalent. Inflammatory cells were present in $126(95.5 \%)$ of the cases. The main diagnosis was benign cellular changes $(122-92.5 \%)$ and atypical squamous cells of undetermined significance, ASC-US, 6 (4.5\%). Were performed 7 (5.3\%) biopsies, being 3 (42.9\%) cervical polyps and $4(57.1 \%)$ nonspecific chronic cervicitis.
Conclusion Despite difficulties to access health care, health indicators were better than expected. There was good cytological coverage and no high-grade lesions were found.

Disclosures Authors declare no conflict of interest.

\section{A CLINICAL STUDY ON THE APPLICATION OF 3D- PRINTING MINIMALLY INVASIVE-GUIDED TEMPLATE IN BRACHYTHERAPY OF PATIENTS WITH LOCALLY ADVANCED CERVICAL CANCER}

Xiangkun Yuan, Yongxia Zhang, Junjun Miao. Hebei Province Cangzhou Hospital of Integrated Traditional and Western Medicine

\subsection{6/ijgc-2020-ESG0.13}

Introduction/Background To explore the clinical application of three-dimensional (3D)-printing minimally invasive-guided template in brachytherapy of patients with locally advanced cervical cancer.

Methodology From May 2016 to December 2018, 59 patients (age, 23-78 years old; median age, 57 years old) with locally advanced cervical cancer. All patients were treated with radical radiotherapy, in which external irradiation was performed, and 3D conformal and intensitymodulated radiotherapy was carried out with a radiation dose of $45 \mathrm{~Gy}$ in 25 fractions of 1.8 Gy per day. The included patients were randomly divided into 2 groups according to random number table method. In the template group, 29 patients assisted by $3 \mathrm{D}$-printing templates to place intrauterine tubes and implant for insertion of needles. In the free implantation group, 30 patients were assisted with freehand implanted intrauterine tubes and implant needles. All patients underwent Computed Tomography (CT) to adjust the position and depth of the insertion needle, and the final CT image was transmitted to the Oncentra brachytherapy planning system, to outline the target area and organs at risk, make treatment plans, and perform treatment.

Results A total of 283 times of combination of intra-luminal and interstitial insertion radiotherapy were undertaken, and a total of 283 times of post-loading radiotherapy plan were formulated, including 141 times in template group and 142 times in free insertion radiotherapy. Complete Response (CR) rate in the template group $(24 / 28 ; 85.71 \%)$ was slightly higher than that in the free transplantation group (22/28; $78.57 \%)$. There was no significant difference in short-term efficacy between the two groups $(z=-0.692, P>0.05)$. Importantly, D90 (90\% of the target volume) of High-Risk Clinical Target Volume (HR-CTV) and Intermediate-Risk Clinical Target Volume (IR-CTV) in the template group were significantly higher than those in the free implantation group $(\mathrm{t}=$ $3.42,2.13, \mathrm{P}<0.05)$. D2 $\mathrm{cm}^{3}$ of bladder, rectum and sigmoid colon was significantly reduced $(\mathrm{t}=-2.59,-4.22,-2.01$, $\mathrm{P}<0.05)$. Therefore, the incidence of grade 1,2 and 3 acute radiation proctitis in the template group was noticeably lower than that in the free transplantation group $(z=-2.112$, $\mathrm{P}<0.05)$. However, there was no significant difference in the incidence of acute radioactive cystitis between the template group and the free implantation group $(\mathrm{z}=-1.686, \mathrm{P}>$ 0.05).

Conclusion For large-block or eccentric cervical cancer, application of the 3D-printing minimally invasive-guided template in brachytherapy of patients with locally advanced cervical 